\title{
O Brasil cumprirá o ODS 3.1 da Agenda 2030? Uma análise sobre a mortalidade materna, de 1996 a 2018
}

\author{
Will Brazil comply with the SDG 3.1 of the 2030 Agenda? \\ An analysis of maternal mortality, from 1996 to 2018
}

Caio Tavares Motta (https://orcid.org/0000-0001-7288-8350) ${ }^{1}$

Marcelo Rasga Moreira (https://orcid.org/0000-0003-3356-7153) ${ }^{1}$

${ }^{1}$ Departamento de Ciências Sociais, Escola Nacional de Saúde Pública Sergio Arouca, Fundação Oswaldo Cruz. R. Leopoldo Bulhões 1.480, Manguinhos. 21041210 Rio de Janeiro RJ Brasil. motta.caio.t@gmail.com

\begin{abstract}
This article aims to analyze if it is possible for Brazil to meet the Sustainable Development Goals (SDG) 3.1, based on a diagnosis of the situation of maternal mortality in the Health Regions (HRs) of Brazil, in 2018, and the main characteristics of this mortality between 1996 and 2018 in the country. The study consists of two articulated phases: (i) bibliographical analysis of maternal mortality in Brazil; (ii) stu$d y$ in the Mortality Information System (SIM, in Portuguese). In 2018, from the 450 HRs, 159 showed a maternal mortality rate (MMR) of above 70 per 100,000 live births (LBs). Between 1996 and 2018, in Brazil, there was a reduction among women 30 to 49 years of age. However, in the age group of 10 to 29 years, there was no change during the time studied. The dissemination of the Maternal Mortality Committees, the PHPN, the PNAISM, and the "Stork Network" have all contributed to improvements in late pregnancies; however, they were inefficient at preventing deaths among young mothers. Compliance with SDG 3.1 requires: prioritization of CIR with MMR greater than 70.0/100,000 LB; qualification of prenatal services, focusing on care among women aged 10 to 29 years and hypertensive complications; and legalization of abortion.
\end{abstract}

Key words Maternal mortality, Policies, SDG
Resumo O objetivo deste artigo é analisar as possibilidades de cumprimento da Meta 3.1 dos Objetivos de Desenvolvimento Sustentável pelo Brasil, a partir do diagnóstico sobre a situação da mortalidade materna nas Regiões de Saúde (CIR), em 2018, e as principais características desta mortalidade, entre 1996 e 2018, no país. Estudo com duas etapas articuladas: análise bibliográfica sobre a mortalidade materna no Brasil; e pesquisa no Sistema de Informação sobre Mortalidade (SIM). Em 2018, das 450 CIR, 159 apresentaram razão de mortalidade materna (RMM) superior a 70 por 100 mil nascidos vivos (NV). Entre 1996 e 2018, no Brasil, houve redução entre mulheres de 30 a 49 anos. Entretanto, a faixa de 10 a 29 anos permaneceu inalterada ao longo da série. A disseminação dos Comitês de Mortalidade Materna, o Programa de Humanização do Pré-Natal e Nascimento (PHPN), a Política Nacional de Atenção Integral à Saúde da Mulher (PNAISM) e a Rede Cegonha influenciaram melhorias na gravidez tardia, mas, não impactaram a prevenção dos óbitos entre mães jovens. O cumprimento do ODS 3.1 requer: a priorização das CIR com RMM superior a 70,0/100 mil NV; a qualificação dos serviços de pré-natal, com foco na atenção entre mulheres de 10 e 29 anos e nas complicações hipertensivas; a e legalização do aborto.

Palavras-chave Mortalidade materna, Politicas, ODS 


\section{Introdução}

A redução da mortalidade materna é um desafio global aos sistemas de saúde. Este compromisso está presente na agenda da Organização das Nações Unidas (ONU), desde os Objetivos de Desenvolvimento do Milênio (ODM), vigente de 2000 a 2015.

Entre as metas dos ODM, a redução da mortalidade materna não foi atingida por muitos países, inclusive o Brasil, permanecendo como parte de uma agenda inconclusa.

Em 2015, é ratificada a nova agenda global: os Objetivos de Desenvolvimento Sustentável (ODS). Dentre estes Objetivos, a redução da mortalidade materna é repactuada na Meta ODS 3.1: até 2030, reduzir a taxa de mortalidade materna global para menos de 70 mortes por 100.000 nascidos vivos ${ }^{1}$.

Com vistas a tornar os ODS mais pertinentes aos desafios locais, o Governo Federal, em parceria com o Instituto de Pesquisa Econômica Aplicada (Ipea), adaptou as Metas da Agenda 2030 à realidade nacional, redefinindo o ODS 3.1: até 2030, reduzir a razão de mortalidade materna para no máximo 30 mortes por 100 mil nascidos vivos ${ }^{2}$.

Apesar do entendimento de que o Brasil tem condições de ir além dos desafios propostos pela ONU, a mortalidade materna no país apresenta distribuição capilarizada em todo o território nacional, com intensa desigualdade dentro e foras das Unidades da Federação.

Tais desigualdades tornam-se ainda mais explícitas quando se examina a realidade brasileira a partir das suas 450 Regiões de Saúde (CIR) espaços geográficos contínuos, formados pela união de municípios limítrofes, de acordo com identidades culturais, sociais e econômicas, que compartilham redes de comunicação e infraestrutura ${ }^{3}$.

Em 2018, das 450 CIR, 356 registraram mortes por causas maternas $(79,1 \%)$. Destas, 313 apresentam razão superior à 30 mortes por 100 mil nascidos vivos (69,5\%), sendo que, 159 possuem razão superior à 70 mortes por 100.000 nascidos vivos $(35,3 \%)^{4}$.

A compreensão desta mortalidade exige uma abordagem que leve em consideração, tantos as desigualdades regionais, quanto questões como: qualidade da informação disponibilizada; políticas, programas e ações voltados para o seu enfrentamento da mortalidade materna; cuidados relacionados ao ciclo gravídico-puerperal; o perfil social e fatores de risco associados a estas mulheres; e aborto ilegal.
A fim de contribuir com este debate, tem-se como objetivo analisar as possibilidades de cumprimento da meta ODS 3.1 pelo Brasil. Para isso, será realizado um diagnóstico sobre (1) a situação da mortalidade materna, com foco nas Regiões de Saúde (CIR), no ano de 2018; e (2) as principais características desta mortalidade, no período de 1996 a 2018.

\section{Aspectos metodológicos}

A pesquisa foi realizada em duas etapas articuladas. Na primeira, foi produzida uma análise bibliográfica sobre a mortalidade materna no Brasil. Na segunda, realizou-se um estudo sobre a mortalidade materna no Sistema de Informação sobre Mortalidade (SIM). A proposta é que a primeira etapa produza o referencial teórico para a análise dos dados levantados na segunda.

A análise bibliográfica teve como foco a produção científica publicada na base SciELO. A pesquisa, realizada em 15 de outubro de 2019, adotou "mortalidade materna" como termo pesquisado e o índice "resumo" como abrangência da pesquisa. Foram encontrados 430 artigos, cujos resumos foram analisados. Com esta análise, 209 artigos foram eliminados: 122 estavam duplicados; 36 tinham como objetivo a mortalidade infantil; 17 referiam-se à mortalidade materna em outros países; sete tratavam da amamentação; e 27 tinham como objeto de estudo a prenhez da rata albina, nascimento na população de bovinos, desempenho reprodutivo de ovelhas e suinos.

Assim, 221 referências estão relacionadas ao objetivo do presente artigo e constituem o universo a ser trabalhado na análise bibliográfica.

Com relação ao estudo realizado no SIM, com base nos óbitos maternos declarados, foi adotado como corte temporal os anos de 1996 a 2018, que abrange todo o período em que o SIM codifica suas informações a partir da CID-10.

Além disso, o ano de 2018, com dados disponíveis mais atuais, foi analisado individualmente, com foco nas Regiões de Saúde (CIR), para investigar as disparidades regionais da mortalidade materna.

A caracterização da mortalidade materna ${ }^{5}$ foi feita a partir dos códigos da CID-10:

- O00-O99 - referentes à Gravidez, Parto e Puerpério (Cap. XV), exceto os códigos O96 e O97;

- A34 - tétano obstétrico (Cap. I);

- B20-B24 - vírus da imunodeficiência humana (Cap. I); 
- D39 - neoplasia de comportamento incerto dos órgãos genitais femininos (Cap. II);

- E23 - hipofunção e outros transtornos da hipófise (Cap. IV);

- F53 - transtornos mentais e comportamentais associados ao puerpério, não classificados em outra parte (Cap. V); e

- M83 - osteomalácia do adulto (Cap. XII).

Para os códigos de fora do Capítulo XV, foi utilizado como filtro de busca o campo "Morte grav/puerp", selecionando as categorias "Durante a gravidez, parto ou aborto" e "Durante o puerpério, até 42 dias".

Apesar do SIM possuir uma cobertura adequada, as mortes maternas são, historicamente, mal informadas nas Declarações de Óbito, no Brasil. A falta de confirmação destas mortes faz com que parte delas sejam classificadas em outras rubricas da CID-10, gerando uma subnumeração.

Além das fragilidades das bases de dados governamentais, este estudo possui como limites: (1) a não utilização do fator de correção para o sub registro das estimativas da mortalidade materna e (2) a não utilização de análises estatísticas de tendências para séries temporais, focado apenas na análise descritiva dos dados.

Os resultados da pesquisa são apresentados em frequências absoluta e relativa e em razão de mortalidade materna (RMM), padronizada por 100 mil nascidos vivos (NV). As variáveis utilizadas para caracterizar o óbito materno foram: Faixa etária, Local de ocorrência, Tipo de causa obstétrica, Grupos CID-10, Categorias CID-10, Escolaridade, Cor e Raça, Estado civil, Unidades da Federação (UF) e Regiões de Saúde (CIR).

\section{Mortalidade materna no Brasil: uma análise bibliográfica}

Dos 221 artigos selecionados para esta análise, $181(81,9 \%)$ foram publicados em periódicos de três áreas específicas: Saúde Pública (104 artigos, 45,1\% do total), Ginecologia e Obstetrícia (42, 19,0\%) e Enfermagem (35, 15,8\%).

Os outros 40 artigos $(18,1 \%)$ são provenientes da História da Saúde (3), Demografia (1) e outras especialidades médicas (36).

Quatro periódicos se destacam pelo volume de publicações no tema: Revista Brasileira de Ginecologia e Obstetrícia (42; 19,0\%); Cadernos de Saúde Pública (34; 15,4\%); Revista de Saúde Pública (22; 9,9\%); e Revista Brasileira de Epidemiologia (17; 7,7\%). Somados, alcançam 115 dos artigos selecionados nesta análise (52,0\%).
$\mathrm{O}$ artigo mais antigo desta análise foi publicado em 1972. Entretanto, foi apenas no final da década de 1990 que a produção científica sobre o tema se intensificou: $92,7 \%$ dos artigos desta análise foram publicados entre 1998 e 2019. Destaca-se o triênio 2016-2017-2018, com 46 publicações (20,8\%).

A seguir, a análise bibliográfica discutirá a definição e classificação do conceito de morte materna e a confiabilidade das informações; as políticas públicas para o enfrentamento da mortalidade materna, voltadas para a atenção e cuidado do ciclo gravídico-puerperal - pré-natal, hospitalização do parto e cesárea; e perfil social e fatores de risco associados ao óbito materno adequação do pré-natal, hipertensão, faixa-etária, escolaridade, raça/cor, estado civil, pré-natal e aborto inseguro.

\section{Análise bibliográfica}

A morte materna é definida, pela Organização Mundial da Saúde (OMS), como o óbito de uma mulher durante a gestação ou durante o período de 42 dias após seu término, devido a qualquer causa relacionada ou agravada pela gravidez ou por medidas relacionadas a ela, exceto por causas acidentais ou incidentais ${ }^{6}$.

A razão de mortalidade materna é o indicador de saúde utilizado para aferir ${ }^{6,7}$ : o risco de morte na gravidez, parto e puerpério; as condições de saúde da população feminina; a adequação do sistema em responder às necessidades sanitárias das mulheres; e as desigualdades sociais.

As mortes maternas são classificadas em: obstétricas diretas, quando estão relacionadas às complicações no ciclo gravídico-puerperal, devido a práticas inadequadas ou omissões; obstétricas indiretas, quando resultam de doenças preexistentes ou que se desenvolveram / agravaram em razão da gravidez; e não obstétricas, quando ocorrem por causas acidentais ou incidentais ${ }^{7-9}$.

As mortes maternas no Brasil são, historicamente, mal declaradas nos atestados de óbitos ${ }^{9}$. A má qualidade da informação está relacionada a erros: na declaração da causa da morte pelos médicos; no preenchimento da Declaração de Óbito; e na seleção da causa básica (subdeclaração) e ausência de declaração de óbito (sub registro) pelos codificadores ${ }^{10}$.

Apesar dessas dificuldades, diversas iniciativas foram tomadas visando a melhoria da qualidade da informação: (1) implantação do Sistema Nacional de Nascidos Vivos ${ }^{6}$, em 1990; (2) a introdução de variáveis de preenchimento obrigatório na declaração de óbito, relacionadas à presença 
de gravidez no momento da morte ${ }^{6,10}$, em 1996; (3) regulamentação da lei de gratuidade dos registros civis de nascimento e óbito ${ }^{6,7}$, em 1998; e (4) atuação dos Comitês de Mortalidade Mater$\mathrm{na}^{7}$.

Além dessas medidas para a melhoria da qualidade da informação, outras políticas foram implementadas, desde a década de 1980, para o enfrentamento da mortalidade materna, tomando-a como um problema de saúde pública e de saúde da mulher.

Em 1983, o Brasil implementou o Programa de Assistência Integral à Saúde da Mulher (PAISM). Marco histórico, o PAISM inaugurou o conceito de "atenção integral à saúde da mulher" e incorporou o planejamento familiar como direito à saúde ${ }^{11,12}$.

Em 1987, foi criado o Comitê de Mortalidade Materna no Estado de São Paulo, com ações estratégicas voltadas para a promoção e prevenção dos óbitos maternos ${ }^{13}$. Com ampla disseminação pelo país, já em 2005, o Brasil contava com: 27 comitês estaduais, 172 regionais, 748 municipais e 206 hospitalares $^{14}$.

No ano 2000, o Ministério da Saúde (MS) implementou o Programa de Humanização do Pré-Natal e Nascimento (PHPN), instituindo procedimentos adequados ao pré-natal, parto e nascimento.

Entre 1990 e 2001, a razão de mortalidade materna decresceu de 141,0 para $80,0 / 100$ mil $\mathrm{NV}^{11,15}$. Tais políticas podem ter impulsionado estes avanços.

Neste período, os países membros da ONU ratificaram os ODM, que pretendiam, dentre outras coisas, reduzir a mortalidade materna em três quartos, até $2015^{11,15}$.

Em 2004, o MS implementou a Política Nacional de Atenção Integral à Saúde da Mulher (PNAISM), reconhecendo a diversidade de grupos de mulheres e de ciclos de vida. Outra política implementada, a Rede Cegonha, em 2011, buscou assegurar o direito ao planejamento reprodutivo, a atenção humanizada e o atendimento seguro durante o ciclo gravídico-puerperal ${ }^{11,12}$.

Apesar disso, o Brasil não atingiu a redução projetada com os ODM: estimava-se um patamar de mortalidade materna inferior a 36,0/100.000 $\mathrm{NV}^{15}$. Entretanto, em 2015, registrou-se uma RMM de 59,7/100.000 N.V, com tendência de estagnação.

Para Leal et al. ${ }^{12}$, a estagnação da razão de mortalidade materna ao longo dos anos 2000 deve-se: à ilegalidade do aborto; aos problemas na qualidade da atenção à gestação, parto e nas- cimento, com desigualdades regionais no provimento de serviços qualificados; à peregrinação das gestantes entre maternidades e serviços; e à epidemia de cesariana e prematuridade.

Tal estagnação é explicada, por Rattner ${ }^{16}$, devido à inadequação da qualidade da atenção à gestação, parto e pós-parto.

A associação entre parto em instituição de saúde e atenção profissional capacitada é considerada a intervenção mais eficaz na redução da morbimortalidade materna: capacitação profissional associada a instalações adequadas possibilitam uma redução de até um terço dos óbitos maternos ${ }^{17}$.

Em 2015, 98,5\% das mulheres tiveram seus filhos em instituições de saúde. Entretanto, a vinculação da gestante a um hospital ainda é considerada inadequada. Nas Regiões Norte e Nordeste, $16 \%$ das mulheres buscaram assistência em mais de uma maternidade até serem atendidas ${ }^{12}$.

A hipermedicalização do parto no Brasil tem sido associada ao aumento da morbidade materna e infantil: $56,9 \%$ dos partos realizados no Brasil foram cirúrgicos, em $2015^{18}$. Ressalta-se que a OMS não reconhece benefícios para a mãe e o bebê em taxas de cesariana acima de $10,0 \%{ }^{12}$.

A assistência pré-natal, por sua vez, é considerada uma intervenção benéfica na prevenção e tratamento para a anemia materna, a hipertensão gestacional, a pré-eclâmpsia grave e eclampsia e os vários tipos de infecções intercorrentes na gestação e parto ${ }^{19}$.

Estudos $^{12}$ mostram que, em 2015, 98,0\% das mães receberam algum tipo de atenção pré-natal. Houve um aumento de $49,0 \%$ para $67,0 \%$ entre a cobertura de sete ou mais consultas, entre 1995 e 2015. Entretanto, as diferenças regionais persistem no país: enquanto a Região Norte registrou 47,0\%, a Região Sul atingiu 80,0\%, em 2015.

A qualidade precária dos serviços de saúde, a falta de integração de cuidados pré-natal e assistência ao parto e o aumento das cesarianas injustificadas ${ }^{20}$ são possíveis explicações para este descompasso entre o aumento do acesso ao pré-natal e a persistência de elevadas razões de mortalidade materna.

É importante assinalar que a inadequação do acompanhamento pré-natal é um importante fator de risco, tanto para morte materna, quanto ao óbito neonatal ${ }^{21-24}$. A ampliação do acesso a este serviço de qualidade é fundamental para a prevenção de ambas as causas de morte ${ }^{23,24}$.

O acompanhamento pré-natal inadequado está mais associado à gestação precoce. Mães adolescentes tardam em iniciar o acompanha- 
mento, resultando em um atendimento incompleto e inadequado ${ }^{25}$.

Entre as mães com 35 anos ou mais há maior conscientização sobre a importância do acompanhamento pré-natal, pois a gravidez tardia é considerada um fator de risco pois aumenta a probabilidade de comorbidades durante a gestação, com repercussões ao feto e ao recém-nascido ${ }^{25}$.

Apesar do óbito materno ser mais recorrentes em mulheres de 20 a 34 anos, devido à maior frequência da gravidez, o aumento da idade associase ao aumento da incidência de doenças crônicas e ao risco de morbimortalidade materna ${ }^{26-30}$.

Entretanto, a mortalidade materna é evitável em mais de $90,0 \%$ dos $\operatorname{casos}^{31-33}$. Diversos estu$\operatorname{dos}^{31-35}$ evidenciam que, no Brasil, há uma predominância de óbitos maternos por causas diretas. Dentre estas causas, destacam-se os distúrbios hemorrágicos, as síndromes hipertensivas e as infecções pós-parto ${ }^{31,34-37}$.

Dentre as complicações médicas que afetam o período gravídico-puerperal, os distúrbios hipertensivos são os mais relevantes ${ }^{38,39}$. Estima-se que a hipertensão incide em 10,0\% das gestantes, e representa a primeira causa de morte materna, respondendo por até $15,0 \%$ delas.

A eclâmpsia é a forma mais grave dos distúrbios hipertensivos e suas complicações estão ligadas ao binômio materno-fetal e à qualidade da assistência prestada, responsável por, aproximadamente, 50 mil mortes globais/ano ${ }^{39}$.

O estudo realizado por Novo e Gianini ${ }^{39}$, no Conjunto Hospitalar de Sorocaba, entre 1995 e 2005, mostra que houve um declínio significativo nas proporções de pacientes eclâmpticas. Esta redução foi atribuída à implementação do PHPN, com a promoção da assistência pré-natal e de manejos intervencionistas mais seguros em ambientes primários e secundários.

A efetividade da assistência pré-natal na atenção primária, complementada pela atenção secundária aos grupos de risco, é a principal forma de prevenção da maioria das complicações hipertensivas da gestação ${ }^{39}$. Entretanto, em 2012, $60,0 \%$ das gestantes iniciaram o pré-natal tardiamente, após a $12^{\text {a }}$ semana, e 25,0\% não recebeu o número mínimo de seis consultas ${ }^{40}$. Além disso, o grande intervalo entre o último atendimento e o parto; e a investigação inadequada do risco gestacional $^{33,35,37}$, agravam os problemas da inadequação destes serviços.

A baixa escolaridade materna é considerada, também, um fator de risco associado à gravidez ${ }^{37}$. Estudo realizado no Rio de Janeiro, entre 1996 e 2004, evidenciou RMM de 28,0/100 mil NV en- tre as mulheres com nível superior e 164,0/100 mil NV entre as analfabetas ${ }^{33}$. Outras pesquisas apontam maior frequência de casos de morte materna em mulheres com Ensino Fundamental $(52,0 \%)^{31}$, com menos de 4 anos de estudo ${ }^{38}$ ou entre 5 e 8 anos $^{28}$.

A cor/raça é outro fator de risco associado ao óbito materno. Em 2001, em estudo realizado nas capitais brasileiras, mulheres pretas tiveram RMM sete vezes maior do que as mulheres brancas ou pardas, com razões de 275,0/100 mil $\mathrm{NV}$ entre mulheres pretas, $46,0 / 100 \mathrm{mil} \mathrm{NV}$ entre mulheres pardas e 43,0/100 mil NV entre bran$\operatorname{cas}^{33,41}$.

Estudos ainda apontam como fatores de risco a situação conjugal insegura e o abando familiar $^{31,34,37}$; o parto cesáreo prévio ou atual ${ }^{27,29}$; a peregrinação antes do parto ${ }^{27}$ : com incidência quatro vezes maior em quem procurou três ou mais serviço $0^{29}$; a gestação múltipla ${ }^{29,30}$; a hipertensão prévia $^{27,28}$; e antecedentes de aborto ${ }^{27,28}$.

No Brasil, o aborto está entre as cinco causas principais de mortalidade materna $a^{42,43}$. De acordo com o artigo 128 do Código Penal Brasileiro, promulgado em 1940, o aborto é caracterizado como crime contra a vida ${ }^{12,42-44}$. No entanto, é permitido em três situações: risco de vida da gestante; gravidez resultante de estupro ou incesto; $\mathrm{e}$ em caso de anencefalia fetal ${ }^{12,43}$.

No entanto, a Lei não se mostrou eficaz para inibir a prática do aborto, deixando muitas mulheres em situações de risco ${ }^{12,43}$. $\mathrm{Na}$ maioria das vezes, é realizado por pessoas não capacitadas em locais insalubres e sem segurança para atender eventuais complicações ${ }^{42}$. Seu status de ilegalidade dificulta a análise da prevalência e magnitude do aborto, com subnotificações nos registros oficiais, principalmente quando se trata de abortos provocados ${ }^{44}$.

Um inquérito nacional, realizado em 2010, verificou que $15,0 \%$ das mulheres brasileiras, no final da vida reprodutiva, já haviam feito aborto, cerca de $50,0 \%$ delas necessitaram de internação $0^{45}$. O percentual de aborto chega a $22,0 \%$ entre mulheres de 35 e 39 anos $^{12}$.

De acordo com uma pesquisa ${ }^{44}$ realizada em Minas Gerais, entre 2000 e 2011, há concentração nos óbitos por aborto em grupos sociais historicamente vulnerabilizados: mulheres negras (70,5\% dos casos) e de baixa escolaridade $(59,6 \%$ - até 7 anos de estudo). O aborto inseguro é uma prática que reforça as iniquidades em saúde no Brasil.

Portanto, os artigos discutidos nesta seção compõem o referencial teórico que analisará os 
dados sobre mortalidade materna levantados no Sistema de Informação sobre Mortalidade e apresentados a seguir.

Mortalidade materna no Brasil: 1996 a 2018

O Gráfico 1 apresenta a razão de mortalidade materna no Brasil entre 1996 e 2018, contextualizando-a com as taxas de mortalidade geral, de mortalidade feminina e entre mulheres de 10-49 anos. Na série, houve crescimento nas taxas de mortalidade geral e feminina, a partir de 2006; entre mulheres de 10-49 anos, as taxas apresentaram uma leve redução; já as razões de mortalidade materna se mantiveram estagnadas ao longo da série.

O Gráfico 2 apresenta o comportamento das razões de mortalidade materna especificadas em quatro faixas etárias de mulheres em idade reprodutiva. Evidencia-se que, quanto maior a faixa etária da mulher, maior é o risco de morte materna associado.

A razão de mortalidade na faixa de 40-49 anos alcançou o máximo de 379,2, em 1998, e a mínima de 147,7 óbitos por 100 mil NV, em 2014. Apesar da redução significativa, as razões permanecem muito elevadas em mulheres deste grupo etário. Por outro lado, as faixas etárias de 10 a 19 e 20 a 29 anos permaneceram estáticas.

Em seguida, a Tabela 1 apresenta as principais características do óbito materno para as quatro faixas selecionadas, entre 1996 e 2018.

Destaca-se, para todas as faixas etárias, a concentração de óbitos em estabelecimentos de saúde; a predominância de causa obstétrica direta; e a prevalência dos Grupos de Causas "Outras afeç̧ões obstétricas NCOP” e "Edema proteinúr e transt hipert gravid parto puerp".

Entre mulheres de 10 a 29 anos, há maior importância das "Complicações relacionadas predom com o puerpério"; já entre as de 30 a 49 anos, destacam-se as "Complicações do trabalho de parto $e$ do parto".

Chama-nos a atenção o grupo de causas "Gravidez que termina em aborto" que alcançou $8,8 \%$ e $9,1 \%$ em mulheres de 10 a 19 e 20 a 29 anos, respectivamente, sendo a quinta causa mais frequente de morte materna entre estas mulheres, no período.

Ressalta-se que o aborto inseguro, praticado de forma ilegal, é um grave problema de saúde pública e os casos que ocorrem na clandestinidade, nem sempre são devidamente informados como morte materna.

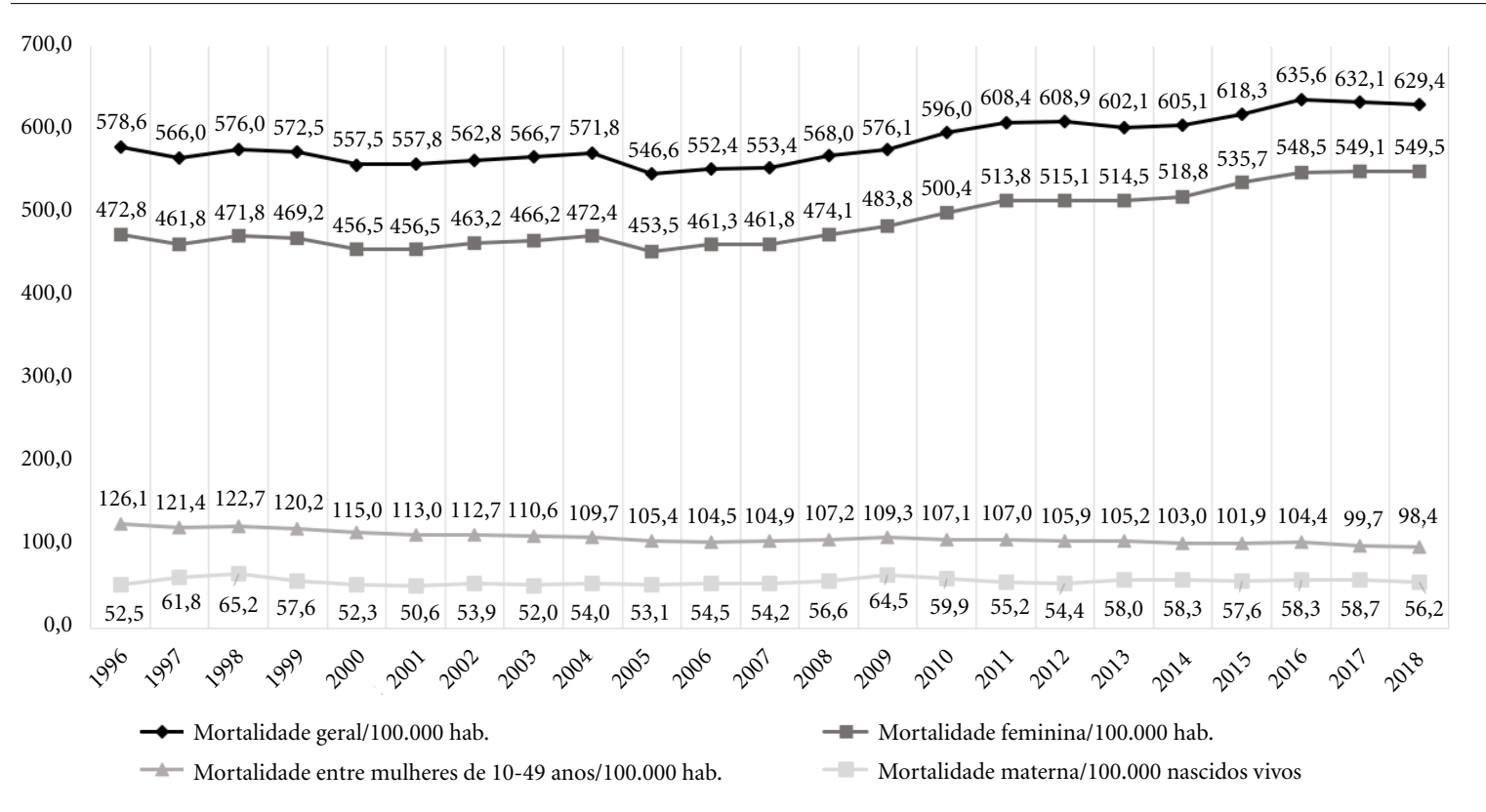

Gráfico 1. Brasil - taxas de mortalidade geral, mortalidade feminina, mortalidade entre mulheres de 10 a 49 anos e razão de mortalidade materna, 1996 a 2018.

Fonte: Elaborado a partir de dados do Sistema de Informação sobre Mortalidade (SIM) e Sistema de Informação sobre Nascidos Vivos (SINASC). 


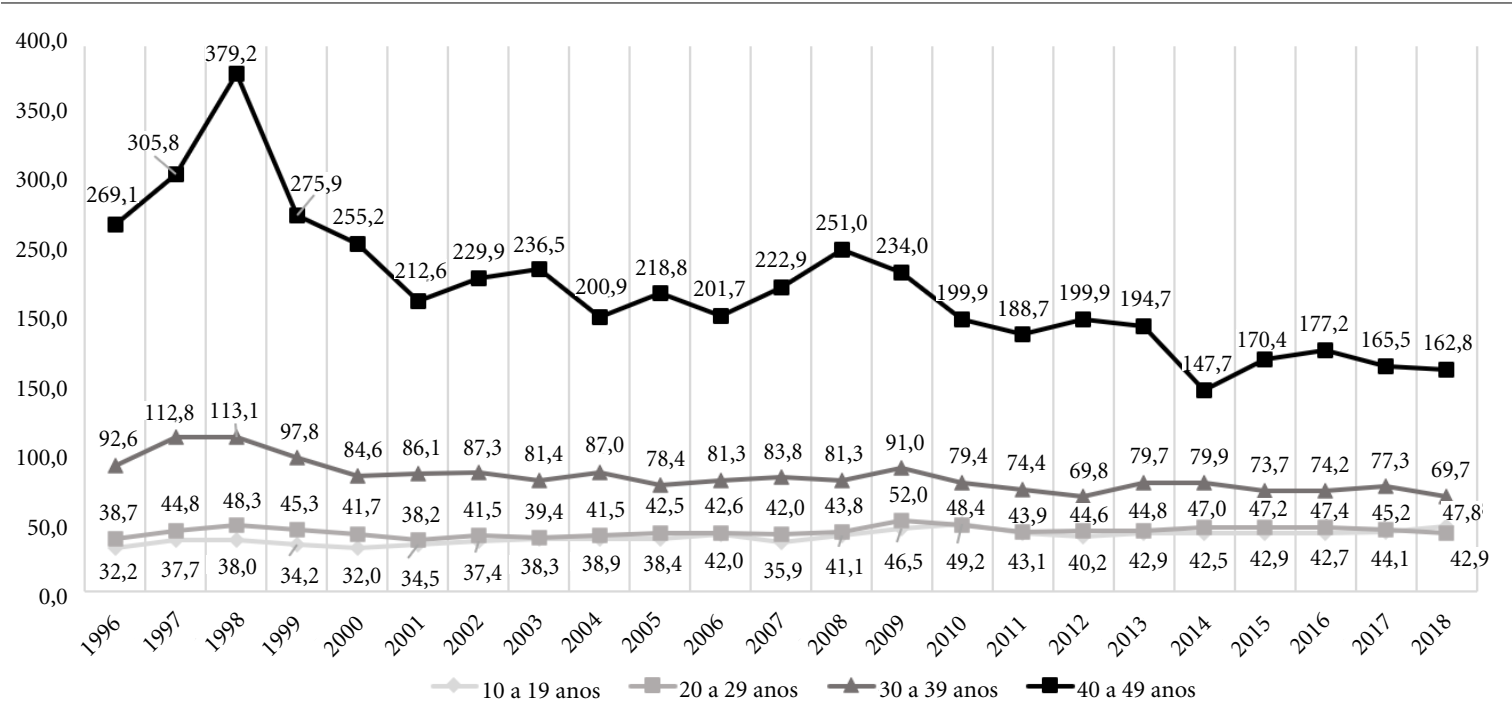

Gráfico 2. Brasil - razão de mortalidade materna entre mulheres de 10 a 49 anos, de 1996 a 2018.

Fonte: Elaborado a partir de dados do Sistema de Informação sobre Mortalidade (SIM) e Sistema de Informação sobre Nascidos Vivos (SINASC).

A Tabela 2 apresenta uma proposta que distribui o conjunto das 450 Regiões de Saúde (CIR) do Brasil em três faixas selecionadas de Razão de Mortalidade Materna: $R M M \leq 30,0 ; 30,1 \leq R M M$ $\leq 70,0$; e $R M M \geq 70,1$.

Assim, em 2018, das 450 CIR, 137 apresentaram $\mathrm{RMM} \leq 30,0 / 100.000 \mathrm{NV}$, inferior à meta ODS 3.1 adaptada pelo Ipea à realidade nacional; por outro lado, 159 delas registraram RMM $\geq 70,1 / 100.000 \mathrm{NV}$, superior à meta ODS 3.1 na versão da ONU.

A construção destas três faixas de RMM se deu a partir de dois parâmetros: 1) a meta ODS 3.1 da ONU, que objetiva reduzir a razão de mortalidade materna global para 70,0/100.000 nascidos vivos $^{1}$; e 2) a readequação da Meta ODS 3.1 à realidade nacional feita pelo Ipea, que visa reduzir a RMM para 30,0/100 mil nascidos vivos ${ }^{2}$. Portanto, os dados apresentados na Tabela 2 buscam organizar as Regiões de Saúde de acordo com tais balizadores.

Por último, o Gráfico 3 organiza as $450 \mathrm{CIR}$, por Unidades da Federação (UF), distribuídas pelas três faixas selecionadas de RMM. Destacase que das 27 UFs, nove apresentaram $50,0 \%$ ou mais de suas respectivas Regiões de Saúde na faixa de RMM superior a 70,1/100 mil nascidos vivos. Dentre elas, quatro são da Região Norte: Ama- zonas (77,8\% das CIR), Amapá (66,7\%), Pará $(53,8 \%)$ e Roraima (50,0\%); três são da Região Nordeste: Maranhão (57,9\%), Ceará $(54,5 \%)$ e Piauí (50,0\%); e dois são da Região Centro-Oeste: Mato Grosso de Sul (75,0\%) e Goiás (55,5\%).

A seguir, os dados apresentados serão examinados a partir do referencial bibliográfico discutido no artigo, com o intuito de analisar as possibilidades de cumprimento da Meta ODS 3.1 pelo Brasil e avaliar se o país cumprirá a meta pactuada.

\section{Discussão}

Convém destacar que o Brasil não atingiu a meta de redução da mortalidade materna pactuada nos ODM. Projetou-se uma RMM de, no máximo, 36,0/100 mil NV ${ }^{15}$ até 2015. O Gráfico 1 evidencia a estagnação da razão de mortalidade materna no Brasil, ao longo dos anos 2000, inviabilizando o cumprimento deste objetivo.

Então, o êxito da Meta ODS 3.1/Agenda 2030 passa pela compreensão dos fatores responsáveis por esta estagnação, para orientar uma ação estratégica sobre estas causas.

No Gráfico 1, apesar da manutenção das altas razões de mortalidade materna, nota-se que, en- 
Tabela 1. Brasil - características da mortalidade materna, a partir das variáveis: local de ocorrência, tipo de causa obstétrica, principais grupos de causas e principais categorias CID-10, escolaridade, raça/cor e estado civil, segundo faixas etárias, 1996- 2018.

\begin{tabular}{|c|c|c|c|c|c|c|c|c|}
\hline \multirow{2}{*}{ Variáveis } & \multicolumn{2}{|c|}{10 a 19 anos } & \multicolumn{2}{|c|}{20 a 29 anos } & \multicolumn{2}{|c|}{30 a 39 anos } & \multicolumn{2}{|c|}{40 a 49 anos } \\
\hline & $\mathbf{n}$ & $\%$ & $\mathbf{n}$ & $\%$ & $\mathbf{n}$ & $\%$ & $\mathbf{n}$ & $\%$ \\
\hline \multicolumn{9}{|l|}{ Local de ocorrência } \\
\hline Hospital/ Estabel. saúde & 5.241 & 93,2 & 14.742 & 93,2 & 12.889 & 92,2 & 2.958 & 90,6 \\
\hline Outros & 380 & 6,8 & 1.079 & 6,8 & 1.084 & 7,8 & 307 & 9,4 \\
\hline \multicolumn{9}{|l|}{ Tipo de causa obstétrica } \\
\hline Direta & 4.123 & 73,3 & 11.005 & 69,6 & 9.682 & 69,3 & 2.123 & 65,1 \\
\hline Indireta & 1.325 & 23,6 & 4.343 & 27,4 & 3.849 & 27,6 & 1.037 & 31,8 \\
\hline Não especificado & 173 & 3,1 & 473 & 3,0 & 439 & 3,1 & 103 & 3,1 \\
\hline \multicolumn{9}{|l|}{ Princip. grupos CID-10 } \\
\hline Outras afecções obstétricas NCOP & 1.379 & 24,5 & 4.159 & 26,3 & 3.500 & 25,1 & 872 & 26,7 \\
\hline $\begin{array}{l}\text { Edema proteinúr e transt. Hipert. Gravid. } \\
\text { parto puerp. }\end{array}$ & 1.423 & 25,3 & 3.533 & 22,3 & 3.123 & 22,4 & 735 & 22,5 \\
\hline $\begin{array}{l}\text { Complicações relacionadas predom. com o } \\
\text { puerpério }\end{array}$ & 721 & 12,8 & 2.321 & 14,7 & 2.537 & 18,2 & 603 & 18,5 \\
\hline $\begin{array}{l}\text { Complicações do trabalho de parto e do } \\
\text { parto }\end{array}$ & 998 & 17,7 & 2.309 & 14,6 & 1.747 & 12,5 & 360 & 11,0 \\
\hline Gravidez que termina em aborto & 496 & 8,8 & 1.434 & 9,1 & 1.039 & 7,4 & 194 & 5,9 \\
\hline \multicolumn{9}{|l|}{ Princip. categorias CID-10 } \\
\hline $\begin{array}{l}\text { O99 Outr. doenc. mat. COP compl. Grav. } \\
\text { parto puerp. }\end{array}$ & 1.037 & 18,6 & 3.309 & 21,5 & 2.810 & 20,8 & 726 & 23,0 \\
\hline O15 Eclâmpsia & 885 & 15,9 & 1.801 & 11,7 & 1.396 & 10,3 & 283 & 9,0 \\
\hline $\begin{array}{l}\text { O14 Hipertensao gestacional c/proteinuria } \\
\text { signif.. }\end{array}$ & 326 & 5,9 & 1.055 & 6,9 & 876 & 6,5 & 175 & 5,5 \\
\hline O72 Hemorragia pós-parto & 230 & 4,1 & 836 & 5,4 & 1.011 & 7,5 & 242 & 7,7 \\
\hline O85 Infecc. puerperal & 453 & 8,1 & 818 & 5,3 & 528 & 3,9 & 112 & 3,5 \\
\hline O88 Embolia orig. obstétrica & 180 & 3,2 & 602 & 3,9 & 598 & 4,4 & 129 & 4,1 \\
\hline O45 Descolamento prematuro da placenta & 138 & 2,5 & 580 & 3,8 & 601 & 4,4 & 166 & 5,3 \\
\hline O62 Anormalidades da contração uterina & 142 & 2,5 & 548 & 3,6 & 633 & 4,7 & 159 & 5,0 \\
\hline O95 Morte obstétrica de causa NE & 173 & 3,1 & 473 & 3,1 & 439 & 3,2 & 103 & 3,3 \\
\hline O06 Aborto NE & 205 & 3,7 & 531 & 3,4 & 339 & 2,5 & 57 & 1,8 \\
\hline \multicolumn{9}{|l|}{ Escolaridade } \\
\hline 0 a 3 anos & 745 & 13,3 & 2.160 & 13,6 & 2.465 & 17,6 & 861 & 26,4 \\
\hline 4 a 11 anos & 2.799 & 49,8 & 7.036 & 44,5 & 5.373 & 38,5 & 974 & 29,8 \\
\hline 12 ou mais & 96 & 1,7 & 918 & 5,8 & 1.248 & 8,9 & 213 & 6,5 \\
\hline Ignorado & 1.981 & 35,2 & 5.707 & 36,1 & 4.887 & 35,0 & 1.217 & 37,3 \\
\hline \multicolumn{9}{|l|}{ Cor e Raça } \\
\hline Branca & 1.565 & 27,8 & 4.941 & 31,2 & 4.903 & 35,1 & 1.031 & 31,6 \\
\hline Preta e parda & 3.082 & 54,8 & 8.291 & 52,4 & 6.851 & 49,0 & 1.628 & 49,9 \\
\hline Amarelo / Indígena & 122 & 2,2 & 215 & 1,4 & 196 & 1,4 & 69 & 2,1 \\
\hline Ignorado & 852 & 15,2 & 2.374 & 15,0 & 2.023 & 14,5 & 537 & 16,4 \\
\hline \multicolumn{9}{|l|}{ Estado Civil } \\
\hline Solteira & 4.159 & 74,0 & 8.606 & 54,4 & 5.597 & 40,1 & 1.088 & 33,3 \\
\hline Casada & 653 & 11,6 & 4.640 & 29,3 & 5.790 & 41,4 & 1.506 & 46,1 \\
\hline Outros & 380 & 6,8 & 1.346 & 8,5 & 1.526 & 10,9 & 425 & 13,0 \\
\hline Ignorado & 429 & 7,6 & 1.229 & 7,8 & 1.060 & 7,6 & 246 & 7,5 \\
\hline Total de óbitos & 5.621 & 100,0 & 15.821 & 100,0 & 13.973 & 100,0 & 3.265 & 100,0 \\
\hline
\end{tabular}


Tabela 2. Mortalidade materna no Brasil em 2018: distribuição por faixas selecionadas de razão de mortalidade materna (RMM), de acordo com o $n^{\circ}$ de Regiões de Saúde (CIR), o somatório do $n^{\circ}$ de óbitos maternos e a razão de mortalidade materna de cada uma das faixas.

\begin{tabular}{|c|c|c|c|c|c|}
\hline \multirow{2}{*}{$\begin{array}{c}\text { Faixas selecionadas por cem mil } \\
\text { nascidos vivos }\end{array}$} & \multicolumn{2}{|c|}{ No de CIR } & \multicolumn{2}{|c|}{$\sum$ do $n^{\circ}$ óbitos maternos } & \multirow{2}{*}{$\begin{array}{l}\text { RMM/100.000 } \\
\text { N.V }\end{array}$} \\
\hline & $\mathbf{N}$ & $\%$ & $\mathbf{N}$ & $\%$ & \\
\hline $\mathrm{RMM} \leq 30,0$ & 137 & 30,4 & 63 & 3,8 & 13,0 \\
\hline $30,1 \leq \mathrm{RMM} \leq 70,0$ & 154 & 34,2 & 811 & 49,0 & 49,6 \\
\hline $\mathrm{RMM} \geq 70,1$ & 159 & 35,3 & 782 & 47,2 & 94,7 \\
\hline Total & 450 & 100,0 & 1.656 & 100,0 & 56,24 \\
\hline
\end{tabular}

Fonte: Elaborada a partir de dados do Sistema de Informação sobre Mortalidade (SIM) e Sistema de Informação sobre Nascidos Vivos (SINASC).

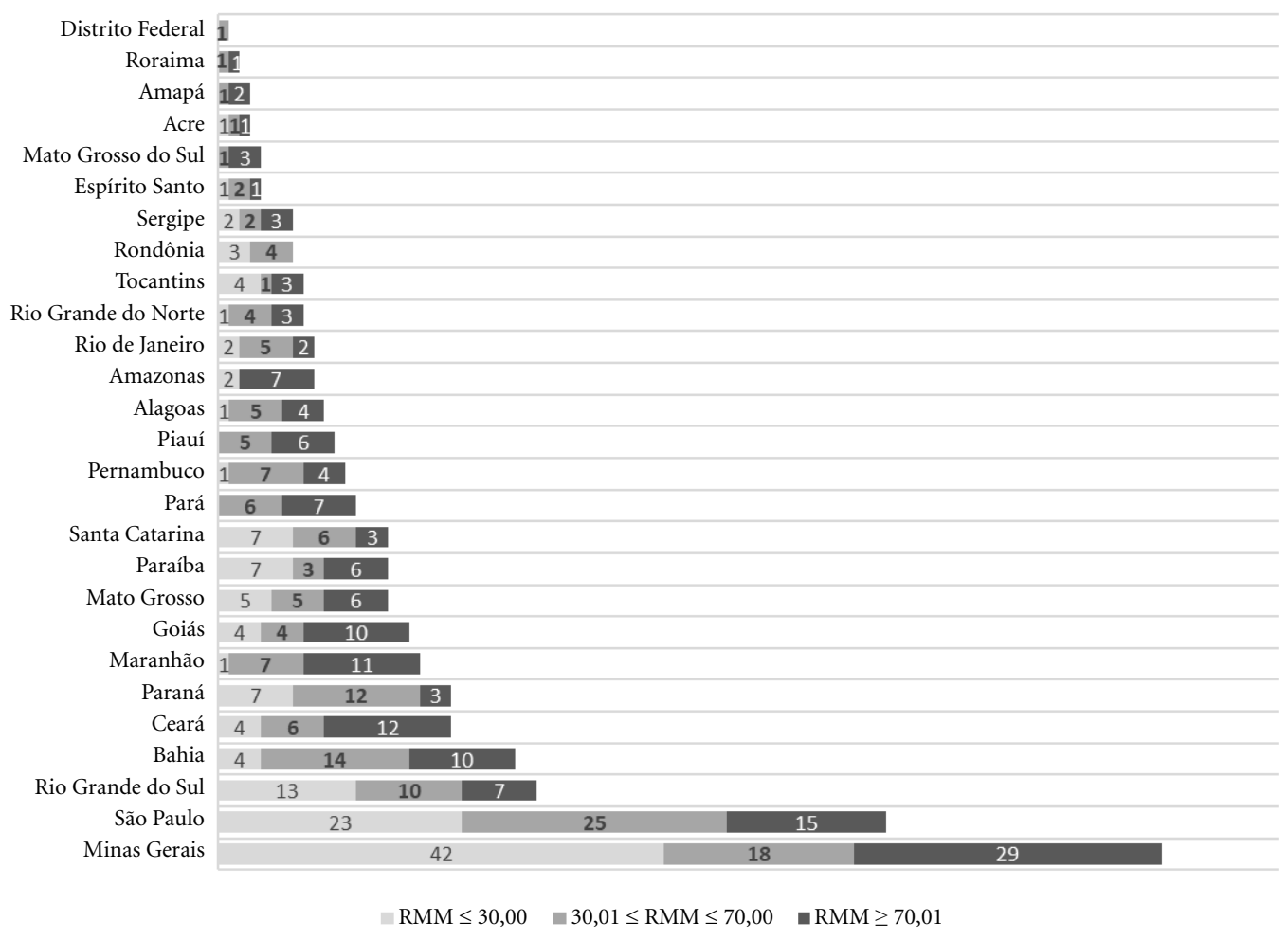

Gráfico 3. Distribuição das Regiões de Saúde (CIR), pelas Unidade da Federação, de acordo com as 3 faixas selecionadas de razão de mortalidade materna: $\mathrm{RMM} \leq 30,0 ; 30,1 \leq \mathrm{RMM} \leq 70,0$; e $\mathrm{RMM} \geq 70,1$. (n = 450)

Fonte: Elaborado a partir de dados do Sistema de Informação sobre Mortalidade (SIM) e Sistema de Informação sobre Nascidos Vivos (SINASC).

tre 1998 e 2001, houve uma redução significativa das razões de 65,2 para 50,6/100.000 NV, redução de $22,4 \%$ na razão.

O Gráfico 2 permite aprofundar o entendimento sobre este declínio: a redução da mortalidade concentrou-se entre as mulheres de 30 a 49 anos, com ênfase na faixa etária de 40 a 49 .
Entre estas, houve redução de 43,9\% neste período (1998 a 2001).

Apesar disso, o Gráfico 2 corrobora a concepção de risco associado à gravidez tardia. A explicação para este fenômeno relaciona-se à associação entre o aumento da idade e o aumento da incidência de doenças crônicas ${ }^{26-30}$. 
Políticas públicas voltadas para o cumprimento da Meta ODS 3.1/Agenda 2030 devem levar em conta a dimensão do risco de óbito materno associado ao aumento da idade, com RMM bastante elevada entre mulheres de 30 a 49 anos.

Ao longo dos anos 2000, um conjunto de políticas voltadas ao enfrentamento da mortalidade materna foram implementadas pelo Ministério da Saúde: a disseminação dos Comitês de Mortalidade Materna; o Programa de Humanização do Pré-Natal e Nascimento (PHPN), em 2000; a Política Nacional de Atenção Integral à Saúde da Mulher (PNAISM), em 2004; e a Rede Cegonha, em 2011.

Estas políticas visaram a promoção da assistência pré-natal e de manejos intervencionistas mais seguros ${ }^{8,9,39}$, o reconhecimento da diversidade de grupos de mulheres e de ciclos de vida, $o$ acesso ao planejamento reprodutivo, a atenção humanizada e o atendimento seguro ao ciclo gravídico-puerperal ${ }^{11,12}$.

À luz destes marcos, depreende-se duas situações dissonantes com o Gráfico 2: as políticas referidas, ao que parece, estimularam reduções importantes entre mulheres de 40 a 49 anos e reduções moderadas entre mulheres de 30 a 39 anos; e entre mulheres de 10 a 29 anos, tais políticas não promoveram reduções na mortalidade materna.

Uma possível explicação para este dado pode estar relacionada à adequação ao pré-natal: entre mulheres acima de 35 anos, há maior conscientização sobre a importância do acompanhamento pré-natal, ao passo que, a inadequação a este serviço está mais associada a gestação precoce, em que mães adolescentes tardam em iniciar o acompanhamento ${ }^{25}$.

Dados do Sistema de Informação sobre Nascidos Vivos (SINASC) ${ }^{46}$ exemplificam esta situação: em 2018, 62,8\% das gestantes entre 10-29 anos tiveram acompanhamento pré-natal considerado adequado/ mais que adequado, enquanto que, entre as mulheres de 30 a 49 anos este percentual de adequação sobe para 74,5\%.

Desse modo, reforçar que mulheres de 10 a 29 anos sejam assistidas adequadamente durante todo o ciclo gravídico puerperal é fundamental para a redução das mortes maternas e, consequentemente, para o cumprimento da Meta ODS 3.1.

Devido à qualidade precária dos serviços de saúde no Brasil, há uma predominância de óbitos maternos por causas diretas ${ }^{31,32,34,35}$, isto é, por complicações no ciclo gravídico-puerperal decorrentes práticas inadequadas ou omissões ${ }^{7-9}$. Esta dimensão foi evidenciada na Tabela 1.
Dentre as mortes obstétricas diretas, os distúrbios hipertensivos são considerados as complicações médicas mais relevantes que afetam o período gravídico-puerperal ${ }^{38,39}$. A efetividade da assistência pré-natal é a principal forma de prevenção destas complicações ${ }^{19,39}$.

A despeito do aumento da adequação do serviço pré-natal (sete consultas ou mais) e da quase universalização das mães que receberam algum nível de acompanhamento $\left(98,0 \%\right.$ em 2015) ${ }^{12}$, pode-se dizer que, o serviço de atenção pré-natal, no Brasil, é ineficiente para prevenir as complicações hipertensivas da gestação.

A partir da Tabela 1 é possível construir um perfil típico da mortalidade materna no Brasil, entre 1996 a 2018: óbito em estabelecimento de saúde $(92,6 \%)$, por causas obstétricas diretas $(69,6 \%)$, sendo os transtornos hipertensivos na gravidez, parto e puerpério sua principal causa $(22,8 \%)$ e a eclampsia sua principal manifestação $(9,0 \%)$; já o perfil social destas mulheres é representado por pretas e pardas $(51,3 \%)$, solteiras (50,3\%), de 4 a 11 anos de escolaridade $(41,8 \%)$.

Estas características da mortalidade materna distribuem-se de forma heterogênea pelo país e produzem desigualdades regionais profundas. A Tabela 2 expressa estas desigualdades a partir de três grupos de Regiões de Saúde (CIR), construídos a partir das faixas selecionadas de mortalidade materna.

As CIR do terceiro grupo apresentam razão de mortalidade materna superior a 70 por 100 mil NV. Neste patamar, dificilmente alcançaram as reduções projetadas pela Meta ODS 3.1 para o país. Este grupo representa mais de um terço das CIR do Brasil.

Tais CIR, juntas, apresentam uma Razão de Mortalidade Materna de 94,7/100 mil NV. Comparativamente, este nível de mortalidade materna assemelha-se às razões registradas, em 2019, nos países: Paraguai $(84,0)$; Micronésia $(88,0)$, Kiribati $(92,0)$, Guatemala $(95,0)$ e Ilhas Salomão $(104,0)^{47}$. Destaca-se que, estes países apresentam Índices de Desenvolvimento Humano (IDH) classificados como médio, já o Brasil apresenta um IDH considerado elevado ${ }^{48}$.

Portanto, para que o Brasil alcance da Meta ODS 3.1 da Agenda 2030, é necessário a correção destas desigualdades internas, por meio da implementação de políticas públicas focadas nestas Regiões de Saúde, cujas razões de mortalidade materna são superiores a 70,1/100 mil NV. Tais políticas devem levar em consideração as especificidades destes territórios e as necessidades sanitárias de mulheres das diferentes regiões. 
O Gráfico 3 possibilitou a identificação de localidades estratégicas para o enfrentamento da mortalidade materna no Brasil formado pelos Estados do Amazonas, Amapá, Pará, Roraima, Maranhão, Ceará, Piauí, Mato Grosso de Sul e Goiás.

Destaca-se que, das 159 CIR com RMM $\geq$ 70,1/100 mil NV, 50,3\% estão nas Regiões Norte e Nordeste. Enquanto que, das 137 CIR com $\mathrm{RMM} \leq 30,0 / 100$ mil NV, 70,4\% estão nas Regiões Sul e Sudeste.

Diante disso, o cumprimento da Meta ODS 3.1 passa pela priorização destas localidades, com a implementação de políticas específicas para este grupo.

\section{Considerações finais}

Em 2018, a RMM do Brasil foi de 56,2/100 mil NV. Isto significa que, para alcançar a redução projetada com a Meta ODS 3.1 da Agenda 2030, adaptada à realidade nacional - 30,0 por 100 mil nascidos vivos, o Brasil deve alcançar uma redução de 46,6\% na RMM, até 2030.

Para isso, as políticas devem levar em conta: 1) a priorização das Regiões de Saúde do Brasil que apresentam RMM superior a 70,00/100.000 nascidos vivos; 2) a prevenção dos óbitos por causas diretas; 3 ) o desenvolvimento de pesquisa nacional para avaliar a qualidade da assistência pré-natal prestada às mulheres; 4) a qualificação dos serviços de pré-natal, com foco na prevenção de complicações hipertensivas; 5) uma maior adequação do acompanhamento pré-natal em mulheres entre 10 e 29 anos; 6) a capacitação das políticas para a prevenção óbitos entre mães jovens; 7) a legalização do aborto; 8) a redução da taxa de cesariana; 9) a redução do risco gestacional associado a gravidez tardia; e 10) melhoria na qualidade da informação sobre mortes maternas.

Trabalhar estas dez recomendações de forma articulada reúne potencial para a formação de um programa que oriente as políticas públicas e propicie o cumprimento da Meta ODS 3.1 da Agenda 2030.

\section{Colaboradores}

CT Motta se responsabilizou pelo levantamento de dados, escrita, análise e revisão final. MR Moreira se responsabilizou pela análise e revisão final. 


\section{Referências}

1. Organização das Nações Unidas (ONU). Transformando nosso mundo: a Agenda 2030 para o Desenvolvimento Sustentável. Nova York: ONU; 2015. [acessado 2020 abr 29]. Disponível em: https://nacoesunidas. org/pos2015/agenda2030/

2. Instituto de Pesquisa Econômica Aplicada (Ipea). ODS - Metas Nacionais dos Objetivos de Desenvolvimento Sustentável. Brasília: Ipea; 2018. [acessado 2020 abr 29]. Disponível em: http://www.ipea.gov. br/portal/images/stories/PDFs/livros/livros/180801_ ods_metas_nac_dos_obj_de_desenv_susten_propos_ de_adequa.pdf

3. Brasil. Ministério da Saúde (MS). Resolução no 1 , de 29 de setembro de 2011. Estabelece diretrizes gerais para a instituição de Regiões de Saúde no âmbito do Sistema Único de Saúde (SUS), nos termos do Decreto $\mathrm{n}^{\circ} 7.508$, de 28 de junho de 2011. Diário Oficial da União 2011; 30 set.

4. Brasil. DATASUS. Sistema de Informação sobre Mortalidade - SIM [acessado 2020 abr 29]. Disponível em: http://www2.datasus.gov.br/DATASUS/index.php?area $=0205 \&$ id $=6937$

5. Brasil. Ministério da Saúde (MS). Guia de Vigilância Epidemiológica do Óbito Materno. Brasília: MS; 2009. [acessado 2020 abr 29]. Disponível em: http://bvsms. saude.gov.br/bvs/publicacoes/guia_vigilancia_epidem_obito_materno.pdf

6. Laurenti Ruy, Mello-Jorge MHP, Gotlieb Sabina Léa Davidson. Reflexões sobre a mensuração da mortalidade materna. Cad Saude Publica 2000; 16(1):23-30.

7. Laurenti R, Mello-Jorge MHP, Gotlieb SLD. A mortalidade materna nas capitais brasileiras: algumas características e estimativa de um fator de ajuste. Rev Bras Epidemiol 2004; 7(4):449-460.

8. Sousa MH, Cecatti JG, Hardy EE, Amaral E, Souza JPD, Serruya S. Sistemas de informação em saúde e monitoramento de morbidade materna grave e mortalidade materna. Rev Bras Saude Mater Infant 2006; 6(2):161-168.

9. Laurenti R. Marcos referenciais para estudos e investigações em mortalidade materna. Rev Saude Publica 1988; 22(6):507-512.

10. Ribeiro CM, Costa AJL, Cascão ÂM, Cavalcanti MLT, Kale PL. Estratégia para seleção e investigação de óbitos de mulheres em idade fértil. Rev Bras Epidemiol 2012; 15(4):725-736.

11. Mamede FV, Prudêncio PS. Contribuições de programas e políticas públicas para a melhora da saúde materna. Rev Gaucha Enferm 2015; 36(n. spe):262-266.

12. Leal MC, Szwarcwald CL, Almeida PVB, Aquino EML, Barreto ML, Barros F, Victora C. Saúde reprodutiva, materna, neonatal e infantil nos 30 anos do Sistema Único de Saúde (SUS). Cien Saude Colet 2018; 23(6):1915-1928.

13. Martins ACS, Silva LS. Perfil epidemiológico de mortalidade materna. Rev Bras Enferm 2018; 71(Supl. 1):677-683.

14. Reis LGC, Pepe VLE, Caetano R. Maternidade segura no Brasil: o longo percurso para a efetivação de um direito. Physis 2011; 21(3):1139-1160.
15. Rodrigues NCP, Monteiro DLM, Almeida AS, Barros MBL, Pereira NA, O’Dwyer G, Andrade MKN, Flynn $\mathrm{MB}$, Lino VTS. Temporal and spatial evolution of maternal and neonatal mortality rates in Brazil, 19972012. J Pediatr (Rio J) 2016; 92(6):567-573.

16. Rattner D. Humanização na atenção a nascimentos e partos: breve referencial teórico. Interface (Botucatu) 2009; 13(Supl. 1):595-602.

17. Cecatti JG, Calderón IMP. Intervenções benéficas durante o parto para a prevenção da mortalidade materna. Rev Bras Ginecol Obstet 2005; 27(6):357-365.

18. Lansky S, Souza KV, Peixoto ERM, Oliveira BJ, Diniz CSG, Vieira NF, Cunha RO, Friche AAL. Violência obstétrica: influência da Exposição Sentidos do Nascer na vivência das gestantes. Cien Saude Colet 2019; 24(8):2811-2824.

19. Calderon IMP, Cecatti JG, Vega CEP. Intervenções benéficas no pré-natal para prevenção da mortalidade materna. Rev Bras Ginecol Obstet 2006; 28(5):310-315.

20. Szwarcwald CL, Escalante JJC, Rabello NDL, Souza Junior PRB, Victora CG. Estimação da razão de mortalidade materna no Brasil, 2008-2011. Cad Saude Publica 2014; 30(Supl. 1):S71-S83.

21. Kassar SB, Melo AMC, Coutinho SB, Lima MC, Lira PC. Fatores de risco para mortalidade neonatal, com especial atenção aos fatores assistenciais relacionados com os cuidados durante o período pré-natal, parto e história reprodutiva materna. J Pediatr (Rio J) 2013; 89(3):269-277.

22. Gaiva MAM, Fujimori E, Sato APS. Maternal and child risk factors associated with neonatal mortality. Texto Contexto Enferm 2016; 25(4):e2290015.

23. Schoeps D, Almeida MF, Alencar GP, França Jr. I, Novaes HMD, Siqueira AAF, Campbell O, Rodrigues LC. Fatores de risco para mortalidade neonatal precoce. Rev Saude Publica 2007; 41(6):1013-1022.

24. Laurenti R, Buchalla CM. Estudo da morbidade e da mortalidade perinatal em maternidades: II - mortalidade perinatal segundo peso ao nascer, idade materna, assistência pré-natal e hábito de fumar da mãe. Rev Saude Publica 1985; 19(3):225-232.

25. Ribeiro FD, Ferrari RAP, Sant'Anna FL, Dalmas JC, Girotto E. Extremos de idade materna e mortalidade infantil: análise entre 2000 e 2009. Rev Paul Pediatr 2014; 32(4):381-388.

26. Leite RMB, Araújo TVB, Albuquerque RM, Andrade ARS, Duarte Neto PJ. Fatores de risco para mortalidade materna em área urbana do Nordeste do Brasil. Cad Saude Publica 2011; 27(10):1977-1985.

27. Silva JMP, Fonseca SC, Dias MAB, Izzo AS, Teixeira GP, Belfort PP. Conceitos, prevalência e características da morbidade materna grave, near miss, no Brasil: revisão sistemática. Rev Bras Saude Mater Infant 2018; 18(1):7-35.

28. Martins AL. Near miss e mulheres negras. Saude Soc 2016; 25(3):573-588.

29. Dias MAB, Domingues RMSM, Schilithz AOC, Nakamura-Pereira M, Diniz CSG, Brum IR et al. Incidência do near miss materno no parto e pós-parto hospitalar: dados da pesquisa Nascer no Brasil. Cad Saude Publica 2014; 30 (Supl. 1):S169-S181. 
30. Moura BLA, Alencar GP, Silva ZP, Almeida MF. Internações por complicações obstétricas na gestação e desfechos maternos e perinatais, em uma coorte de gestantes no Sistema Único de Saúde no Município de São Paulo, Brasil. Cad Saude Publica 2018; 34(1):e00188016.

31. Carreno I, Bonilha ALL, Costa JSD. Evolução temporal e distribuição espacial da morte materna. Rev Saude Publica 2014; 48(4):662-670.

32. Souza ML, Burgardt D, Ferreira LAP, Bub MBC, Monticelli M, Lentz HE. Meninas Catarinas: a vida perdida ao ser mãe. Rev Esc Enferm USP 2010; 44(2):318-323.

33. Morse ML, Fonseca SC, Barbosa MD, Calil MB, Eyer FPC. Mortalidade materna no Brasil: o que mostra a produção científica nos últimos 30 anos? Cad Saude Publica 2011; 27(4):623-638.

34. Riquinho DL, Correia SG. Mortalidade materna: perfil sócio-demográfico e causal. Rev Bras Enferm 2006; 59(3):303-307

35. Lima MRG, Coelho ASF, Salge AKM, Guimarães JV, Costa PS, Sousa TCC, Mattos DV, Sousa MAA. Alterações maternas e desfecho gravídico-puerperal na ocorrência de óbito materno. Cad Saude Colet 2017; 25(3):324-331.

36. Siqueira AAF, Tanaka ACA, Santana RM, Almeida PAM. Mortalidade materna no Brasil, 1980. Rev Saude Publica 1984; 18(6):448-465.

37. Martins ACS, Silva LS. Perfil epidemiológico de mortalidade materna. Rev Bras Enferm 2018; 71(Supl. 1):677-683.

38. Bezerra EHM, Alencar JCA, Feitosa RFG, Carvalho AAA. Mortalidade materna por hipertensão: índice e análise de suas características em uma maternidade -escola. Rev Bras Ginecol Obstet 2005; 27(9):548-553.

39. Novo JLVG, Gianini RJ. Mortalidade materna por eclâmpsia. Rev Bras Saude Mater Infant 2010; 10(2):209-217.

40. Saintrain SV, Oliveira JGR, Saintrain MVL, Bruno ZV, Borges JLN, Daher EF, Silva GBJ. Fatores associados à morte materna em unidade de terapia intensiva. Rev Bras Ter Intensiva 2016; 28(4):397-404.

41. Chor D, Lima CRA. Aspectos epidemiológicos das desigualdades raciais em saúde no Brasil. Cad Saude Publica 2005; 21(5):1586-1594.

42. Domingos SRF, Merighi MAB. O aborto como causa de mortalidade materna: um pensar para o cuidado de enfermagem. Esc Anna Nery 2010; 14(1):177-181.

43. Zordo S. Representações e experiências sobre aborto legal e ilegal dos ginecologistas-obstetras trabalhando em dois hospitais maternidade de Salvador da Bahia. Cien Saude Colet 2012; 17(7):1745-1754.

44. Martins EF, Almeida PFB, Paixão CO, Bicalho PG, Errico LSP. Causas múltiplas de mortalidade materna relacionada ao aborto no Estado de Minas Gerais, Brasil, 2000-2011. Cad Saude Publica [periódico na Internet]. 2017 [acessado 2020 jan 12];33(1): [cerca de 10p.]. Disponível em: https://doi.org/10.1590/ 0102-311X00133116.
45. Kale PL, Jorge MHPM, Fonseca SC, Cascão AM, Silva KS, Reis AC, Taniguchi MT. Mortes de mulheres internadas para parto e por aborto e de seus conceptos em maternidades públicas. Cien Saude Colet 2018; 23(5):1577-1590.

46. Brasil. DATASUS. Sistema de Informação sobre Nascidos Vivos - SINASC [acessado 2020 ago 03]. Disponível em: http://tabnet.datasus.gov.br/cgi/tabcgi. exe?sinasc/cnv/nvuf.def

47. World Health Organization (WHO). Trends in Maternal Mortality 2000 to 2017. Geneva: WHO; 2019. [cited 2020 Oct 23]. Available from: https://www.who. int/reproductivehealth/publications/maternal-mortality-2000-2017/en/

48. Programa das Nações Unidas para o Desenvolvimento (PNUD). Relatório do Desenvolvimento Humano 2019. Nova York: PNUD; 2019. [acessado 2020 out 26]. Disponível em: http://hdr.undp.org/sites/default/ files/hdr_2019_pt.pdf
Artigo apresentado em 20/11/2020

Aprovado em 19/11/2021

Versão final apresentada em 21/11/2021

Editores-chefes: Romeu Gomes, Antônio Augusto Moura da Silva 
